\title{
System Optimization and Robustness Stability Control for GIS Inspection Robot in Complex Microgrid Networks
}

\author{
Yu Yan, ${ }^{1}$ Wei Jiang ${ }^{(1)},{ }^{2,3}$ Zhiping Luo, ${ }^{1}$ Jianjun Zhang, ${ }^{1}$ and Weidong Liu ${ }^{1}$ \\ ${ }^{1}$ State Grid Hunan Maintenance Company, Changsha 410004, China \\ ${ }^{2}$ Wuhan Textile University, Hubei Key Laboratory of Digital Textile Equipment, Wuhan 430200, China \\ ${ }^{3}$ Wuhan Textile University, Hubei Engineering Research Center of Industrial Detonator Intelligent Assembly, \\ Wuhan 430073, China \\ Correspondence should be addressed to Wei Jiang; jiangwei2013@whu.edu.cn
}

Received 27 October 2020; Revised 21 November 2020; Accepted 27 January 2021; Published 27 February 2021

Academic Editor: Ruoli Tang

Copyright ( 2021 Yu Yan et al. This is an open access article distributed under the Creative Commons Attribution License, which permits unrestricted use, distribution, and reproduction in any medium, provided the original work is properly cited.

\begin{abstract}
GIS (gas-insulated switchgear) is important equipment in the substation system in a complex microgrid network. Due to the longterm service in harsh operation environments, the electrical performance of GIS equipment will be seriously affected. Therefore, the regular maintenance of GIS equipment in the substation system is a routine task so as to ensure the normal operation of the microgrid networks. The traditional method relies on manual labor, where not only with the low operation efficiency but also regarding some maintenance, labors cannot reach the inside of the GIS equipment, which seriously restricted the completion of GIS equipment maintenance operation. Based on the above analysis, a basic configuration of a GIS equipment maintenance robot with smart structure, convenient control, and stable motion characteristic has been proposed in this paper. Through the optimization design of the chassis adsorption system, the GIS inspection robot can achieve hanging and vertical adsorption inside the GIS equipment and some special motion status. In this way, the entire GIS equipment can be inspected without blind spots. Through establishing the mechanical model of the GIS inspection robot under the hanging and vertical motion state, the robustness and stability of the GIS inspection robot in the special status have been analyzed, and the corresponding GIS inspection robot robust stability motion control method has also been proposed. Finally, through the integrated design of mechanical system and hardware and software control system, the GIS inspection robot physical prototype system has been developed and the maintenance operation experiment has been carried out in Hunan Electric Power Company Maintenance Company through simulating the internal GIS equipment. The physical prototype can realize the basic movement and special movement in internal GIS equipment without any blind spots and complete the inspection task of GIS equipment operation; the movement of GIS robot is dexterous and stable in the whole operation process, which has strong robust stability. The research of GIS inspection robot in the complex microgrid networks has important theoretical significance and practical application value for the intelligent operation and maintenance management of substation system in complex microgrid networks.
\end{abstract}

\section{Introduction}

There are a large number of GIS (gas-insulated switchgear) types of equipment [1-4] built in the power system substation networks, GIS is a metal-enclosed equipment, and once there is an internal fault, the gas chamber needs to be opened for searching and processing, which not only is very inconvenient but also leads to poor safety and economy. In most cases, GIS equipment maintenance cannot directly dismantle the faulty spot. It needs to be dismantled layer by layer, which needs the recovery time, and a small problem may cost so much and take half a month. From the current internal defects, more than $80 \%$ of the internal defects are mainly small problems, such as fixing fitting loose, poor cleanliness, and some other small problems. In order to ensure the safety and reliable and stable operation of the substation network, using GIS inspection robots to assist or even replace manual maintenance of GIS equipment can not only improve working efficiency but also liberate the labors from the dangerous power maintenance environment. In 
order to achieve GIS equipment maintenance without blind spots, the GIS inspection robots should be able to climb along the inside of GIS equipment pipelines. Therefore, the robot climbing ability is the key to GIS equipment maintenance operations. In recent decades, wall-climbing robots [5-8] have been widely used in different fields; it is a special type mobile robot, because its main feature is that it can overcome its own gravity on the surface of the three-dimensional environment and then move to complete the GIS inspection operation. Therefore, the wall-climbing robot not only needs to have the same motion mechanism as the traditional mobile robot but also needs a special adsorption mechanism for the robot to absorb the three-dimensional wall surface. Due to the particularity of the wall-climbing robot motion and the influence of the surface environment, the robot motion control becomes very complicated. Therefore, the research on wall-climbing robots will be more challenging than mobile robots; at the same time, the GIS inspection robots with wall-climbing functions are used to replace humans to complete these high-altitude, high-risk inspection tasks, which can improve working efficiency and protect personnel safety; thus, the research has important theoretical significance and practical application value for the GIS equipment wall-climbing robot in the complex microgrid networks.

GIS inspection robot mainly relies on its climbing ability on the GIS equipment surface; in foreign countries, the research on wall-climbing robots can be divided into three categories [9-11]. The first category is the negative pressurebased wall-climbing robot developed by Miyazaki University in Japan in 1966; the robot is very huge and heavy; after 1990, the wall-climbing robot based on the negative pressure adsorption mechanism was developed rapidly. Although the adsorption method can be adapted to the wall surface of many different materials, it has higher requirements on the wall surface, and the wall surface condition affects the robot motion stability, which limits the development of negative pressure adsorption wall-climbing robots. The second category is the foot-type bionic wall-climbing mode, such as Rise series robot based on bionic adsorption, which was developed by Boston Dynamics, Rise $V_{2}$ is a hexapod bionic wall-climbing robot, the soles of Rise's feet have hairy claws which are similar to those of wall-climbing animals, and it relies on multijoint motion to crawl. Rise has a bionic spine and tail, which can improve the motion balance of the robot on different walls by changing the state of the spine and tail. Rise $V_{3}$ is a four-leg bionic wall-climbing robot based on Rise $V_{2}$, its size is $250 \mathrm{~mm}$ in length, its weight is $2 \mathrm{~kg}$, and the speed is $0.3 \mathrm{~m} / \mathrm{s}$. It is mainly used in search and rescue operations and can be used for reconnaissance and surveillance. The biggest feature of the Rise series [12] robot is that its sole has a special microhair structure adsorption mechanism; through virtue of this mechanism, Rise can realize vertical movement without external force, which greatly reduces the overall power consumption of the robot, and as the structure is very complicated, it crawls very slowly. The third category is Magnebike wall-climbing robot developed by the Federal Institute of Technology Zurich, Switzerland, with a total weight of $3.3 \mathrm{~kg}$ and a volume of
$170 \times 130 \times 220 \mathrm{~mm}^{3}$, with a maximum walking speed of $2.7 \mathrm{~m} / \mathrm{min}$ and a magnetic adsorption force of $250 \mathrm{~N}$, which is mainly used in pipe inspection and other scenarios; the research has important reference value for GIS equipment maintenance operations. Domestically, the first research on wall-climbing robots in China was in Harbin University of Technology, which successfully developed a wall-climbing robot $[13,14]$ called CLR-I in 1994, CLR-I's adsorption method is negative pressure adsorption, with all-directional mobility, and it is able to walk flexibly on the wall surface, but CLR-I uses wired and console connection mode, which is extremely inconvenient to control and operate. Four years later, Harbin University of Technology successfully developed the CLR-II wall-climbing robot on the basis of CLR-I, which aims at the inconvenience of CLR-I cable operation, removing wired control, increasing wireless communication technology, and improving the control convenience. The University of the Chinese Academy of Sciences has also made important contributions to wall-climbing robots and its magnetic adsorption track-type wall-climbing robots $[15,16]$ have optimized the size and structure of the magnetic array, so that the adsorption force reached by the robot is as high as $503 \mathrm{~N}$, which can be well adsorbed to the surface of the conductive magnet and provide a reliable guarantee for the robot motion on the wall surface. However, the tracktype structure causes it to move less flexibly and it cannot adapt to the surface appropriately.

Through the above analysis, it can be concluded that the key for the research of GIS inspection robot is its climbing ability in GIS equipment and the key of its climbing ability is mainly dependent on magnetic adsorption mechanism $[17,18]$ which is to use magnetic force generated between the magnetic source and the conductor magnet, and so as adsorption on the object surface, magnetic sources can be divided into permanent magnet and electromagnetic. Magnetic adsorption form can produce great adsorption force; therefore, magnetic adsorption wall-climbing robot can ignore the impact of surface roughness, which enhances the operational stability $[19,20]$ of the robot system; the main contribution of this paper is to propose a robot climbing mechanism with adsorption function and its stable climbing inspection and application in power GIS equipment. Therefore, in view of the current power GIS maintenance task in the complex microgrid networks, this paper puts forward a kind of GIS equipment maintenance operation by using the wall-climbing robot with adsorption ability, so as to optimize the design of the streamlined mechanical configuration; then the corresponding control system and physical prototype models have been developed through the simulation and analysis of the GIS inspection robot motion robust stability in the process of operation and walking; this research puts forward the corresponding robust control method. Finally, in GIS equipment, the physical prototype has been used to carry out GIS equipment maintenance operation experiments and robot mechanism streamlined and flexible motion, which can achieve GIS equipment maintenance without blind spots; in the operation process, robot moves stably in the complex microgrid networks environment and can resist electromagnetic interference and other external environment 
impacts on the GIS inspection robot; the robot showed a strong robustness. The research of this paper is a new application and exploration for the maintenance of power intelligent microgrid networks; its research and implementation are of great theoretical significance and practical application value to improve the energy efficiency of complex microgrid networks system, promoting the automation and intelligence of power system.

\section{GIS Inspection Robot Mechanical System Design and Optimization}

2.1. The Overall Design of the GIS Inspection Robot System. When GIS inspection robot replaces manual GIS equipment inspection operation, there are three requirements, which are the stable wall adsorption ability, flexible mobility on three-dimensional walls, and adaptive ability to different wall surfaces. In order to meet the three above requirements, this paper designs a wheel mobile robot that can achieve alldirectional climbing inside GIS equipment as shown in Figure 1, where Figure 1(a) is the robot physical prototype operation environment, Figure 1(b) is robot three-dimensional structure, and Figures $1(\mathrm{c})-1(\mathrm{f})$ are the motion planning of robot inspection operations. The robot is highly modular in design and it consists mainly of all-directional mobile chassis module, magnetic adsorption module, sensors, and electrical control system with mass of $2 \mathrm{~kg}$. The robot can be used as a mobile platform, equipped with small detection equipment and manipulator to complete dust removal maintenance function.

The GIS climbing robot designed in this paper needs to move on the wall and carry manipulator equipment to complete the inspection and maintenance work; considering that the tracking scheme is slow and not flexible enough, the control is difficult, and the adsorption method is relatively single, so all-directional wheat wheel can be selected in the mobile scheme, which has both speed and flexibility, and also the control difficulty is relatively low. Climbing robot in the three-dimensional environment needs to have adsorption capacity, and the robot working environment is mostly ferromagnetic material wall surface, so the use of permanent magnet adsorption scheme has been adopted in order to reduce the volume and mass of the magnets, and the use of Halbach magnetic adsorption array as adsorption scheme can enhance adsorption side magnetism.

2.2. GIS Inspection Robot Mobile Chassis Design and Optimization. The current mobile robot moves in three main ways, which are foot mode, track mode, and wheel mode; wallclimbing robots serve as a branch of mobile robots, and these three methods are also suitable for wall-climbing robots. The wall-climbing robot with track-type mobile scheme is the most commonly used, its advantages are stability, high adaptability to the wall, and strong ability to cross the barrier; GIS wallclimbing robot with wheel scheme is also widely used, and the advantages of wheel robot are simple structure, high efficiency, fast speed, and easy control; the disadvantage is that the wall adaptability is lower than those of track-type mode robot and foot mode robot. Foot mode climbing robot usually uses bionic adsorption scheme; the advantage is that the wall-climbing adaptability is very sound, whether it is flat, surface, irregular surface, all can adapted, the ability to cross the barrier is the strongest. The disadvantage is that it is slow and difficult to control, as the robot requires flexible all-directional mobility; from the above discussion, the GIS robot mobile scheme selects a wheel mobile scheme. Therefore, the robot chassis uses a $3 \mathrm{~mm}$ thick carbon fiber plate as a support plate and four McNamee wheels to form an O-shaped all-way chassis, which is shown in Figure 2.

2.3. GIS Inspection Robot Magnetic Adsorption Array Design and Optimization. Three-dimensional wall-climbing ability of GIS robot is on the premise of stable adsorption on the wall surface; the current mainstream adsorption methods include bionic adsorption, negative pressure adsorption, and magnetic adsorption; most industrial productions of pipelines, tanks, and other equipment with curved wall surfaces are made of ferromagnetic materials; this paper adapts magnetic adsorption as a robot adsorption scheme. In order to achieve miniaturization and light weight, magnetic adsorption arrays must be optimized so as to produce large adsorption forces at very small volume and mass. Halbach magnetic arrays are a special form of magnet scheduling which enhances the single magnetic field. The principle is shown in Figure 3, where the longitudinal magnetized magnetic field is shown in Figure 3(a) and the transverse magnetized magnetic field is shown in Figure 3(b), which can theoretically completely cancel out the magnetic field on one side and enhance the magnetic field on the other side; ideally, a complete one-way magnetic field can be obtained.

In the practical applications, the continuous change of magnetic field direction cannot be achieved, usually the magnetic induction direction changes for discrete processing, discrete magnetic blocks at a certain angle of change stitched together to approximate the magnetic induction strength ideal sine changes, the magnetic adsorption array arrangement rules designed in this paper are shown in Figure 4, the super magnetic force of rare Earth $\mathrm{NdFeB}$ magnet has been used, with size of $20 \times 10 \times 10 \mathrm{~mm}$, and the design interval is $1 \mathrm{~mm}$.

Based on the above analysis results, the determined structure of the magnetic array module and its distribution on the platform of GIS inspection robot system is shown in Figure 5; the magnetic array has been constructed according to the pattern of the Halbach array; the whole machine is installed with two adsorption arrays, which distributed on both sides of the chassis and support the carbon plate; in the magnetic body, the wall gap is $5 \mathrm{~mm}$, which can provide nearly $200 \mathrm{~N}$ adsorption force.

\section{GIS Inspection Robot Control System Design and Optimization}

The design of GIS inspection robot control system is the core of the stable operation for the robot whole system, and its hardware configuration level is directly related to the system 


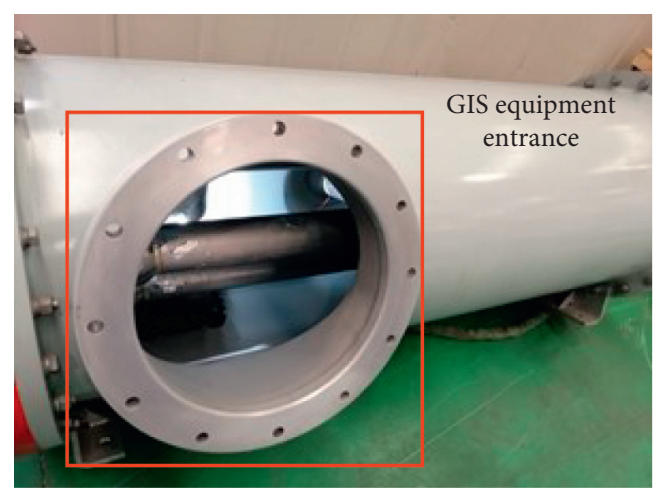

(a)

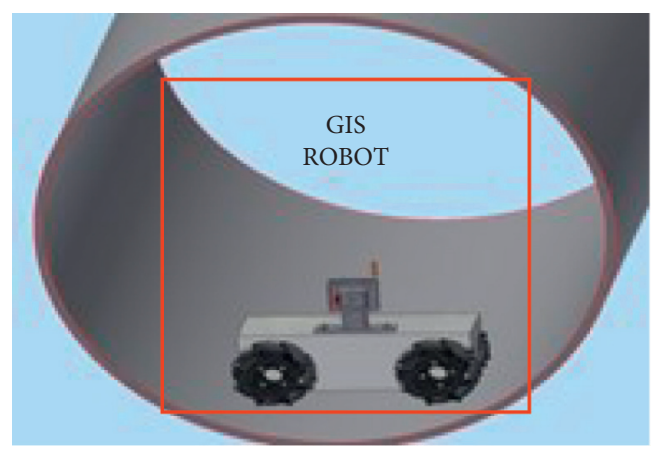

(c)

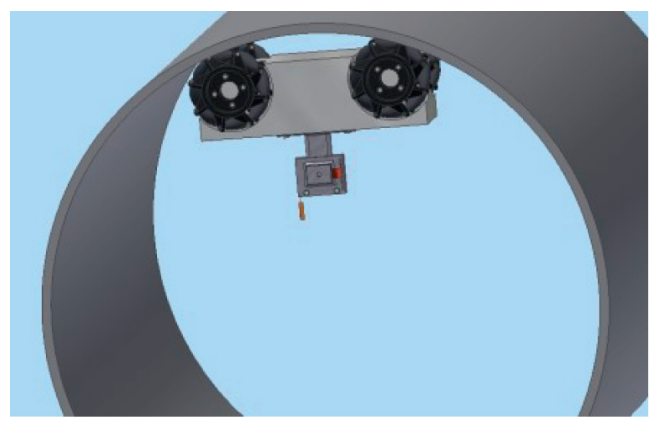

(e)

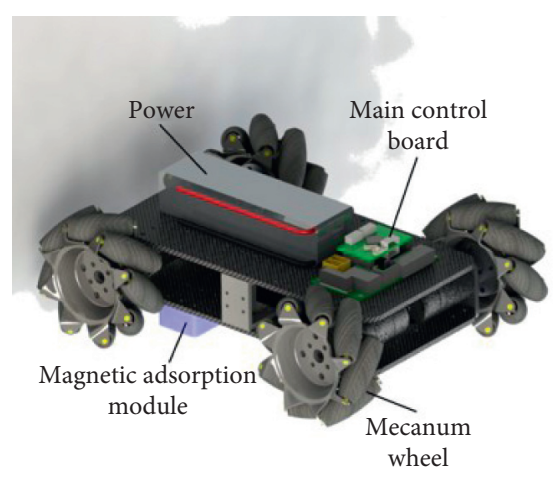

(b)

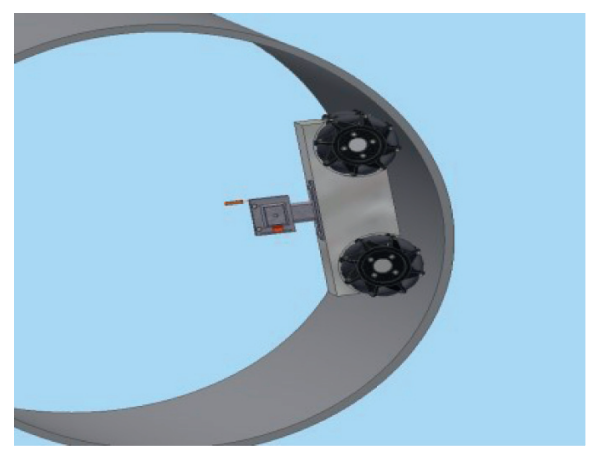

(d)

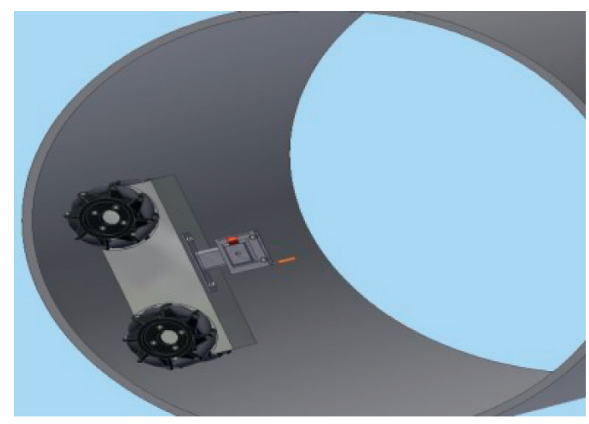

(f)

FIGURE 1: GIS equipment inspection robot with adsorption climbing function. (a) GIS operation environment, (b) robot three-dimensional structure, (c) robot inspection status-1 (initial state), (d) robot inspection status-2 (uphill slope), (e) robot inspection status-3 (apical adsorption), and (f) robot inspection status-4 (downhill slope).

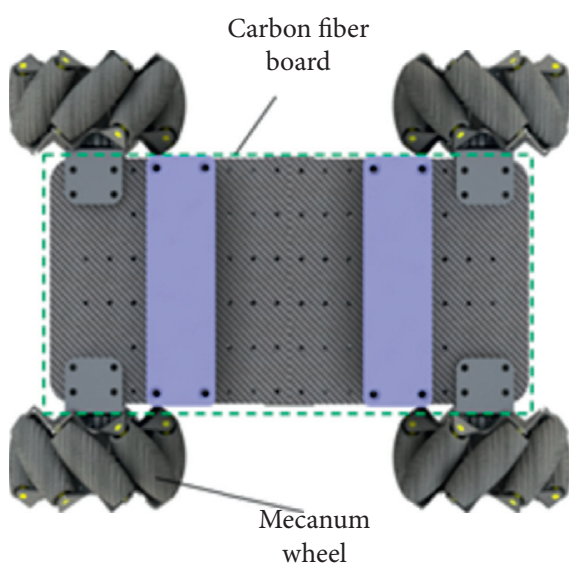

FIGURE 2: GIS equipment inspection robot chassis structure diagram. 


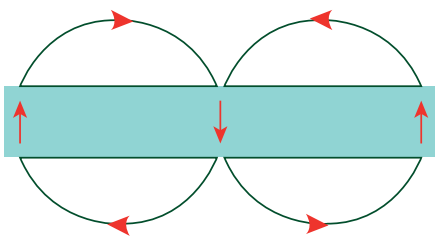

(a)

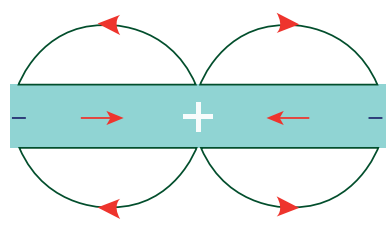

(b)

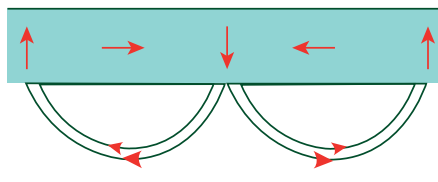

(c)

FIGURE 3: A schematic principle of the Halbach magnetic adsorption array. (a) $m_{x}=\sin k x$; (b) $m_{y}=\cos k x$; (c) $m=m_{x}+m_{y}$.

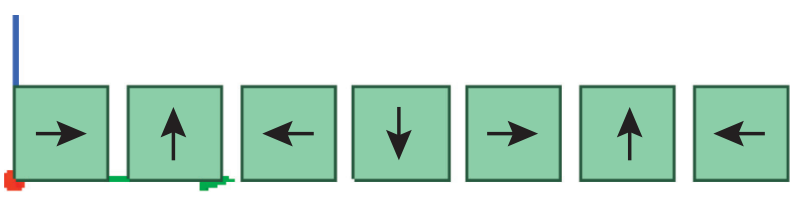

Figure 4: Halbach magnet arrangement rule diagram.

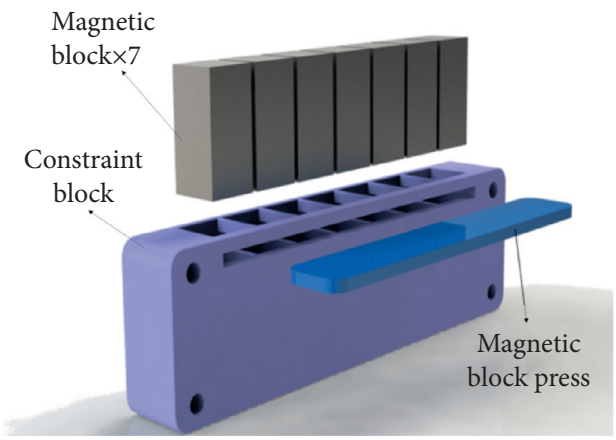

(a)

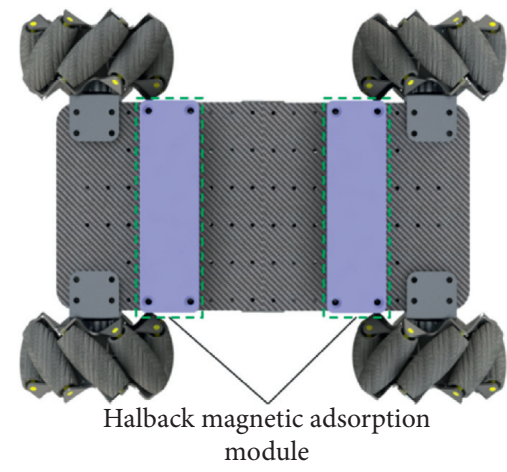

(b)

Figure 5: Magnetic array module. (a) Magnet installation structure illustration; (b) distribution of magnetic array on the chassis.

robustness and intelligence, and the design strategy of the control system also determines the functional characteristics, using scope and scalability of the system. According to the functional requirements and design purposes of the GIS inspection robot, the overall block diagram of the robot control system is shown in Figure 6. MPU is the main core of the robot; communication between the different motors and the CAN bus repeater can be achieved by connecting a CAN bus through which the CAN repeater can drive the four motors. CAN repeaters, environment-aware modules, and IMU modules communicate through a CAN bus and MPU so as to complete the overall control of the robot by the MPU.

The motor drive control algorithm used by the GIS (gasinsulated switchgear) inspection robot is a three-ring nested PID (Proportional Integral Derivative) algorithm, and the corner data read by the angle encoder that the robot drives the motor can be used as input to the position ring. The input value and the robot chassis motion of the MPU wheel corner data in the position ring PID operation, after the position ring have been solved, the output value can be obtained, the corner data collected by the angle encoder that drives the motor can be used as the input value of the speed ring together with the value after the time difference, and an output value can be obtained after the speed ring with PID operation. This output value is then used as an input to the current ring together with the current status generated by the motor driver, and then the PID operation of the current ring can be performed; the final output value is used as the input value of the motor driver. Through the three-ring nested PID algorithm, the purpose of the robot motor accurate motion control can be finally achieved, the main control objects are three typical motion states of GIS inspection robot, and the structure of the control system is shown in Figure 7.

GIS inspection robot uses STM32F405RGT6 chip as a robot MPU module and completes the speed ring and position ring control on the basis of brushless motor regulator; the robot control board operation frequency can reach up to $85 \mathrm{MHz}$; its computing power can fully meet the real-time control needs of the robot according to the robot motion and active adaptation functions, as well as the need for volume and quality control, combined with the position of the robot. The $D_{j i}$ M2006 brushless motor as the driving motor of the chassis and active adaptation body has been selected, which is used in combination with the C610 brushless motor 


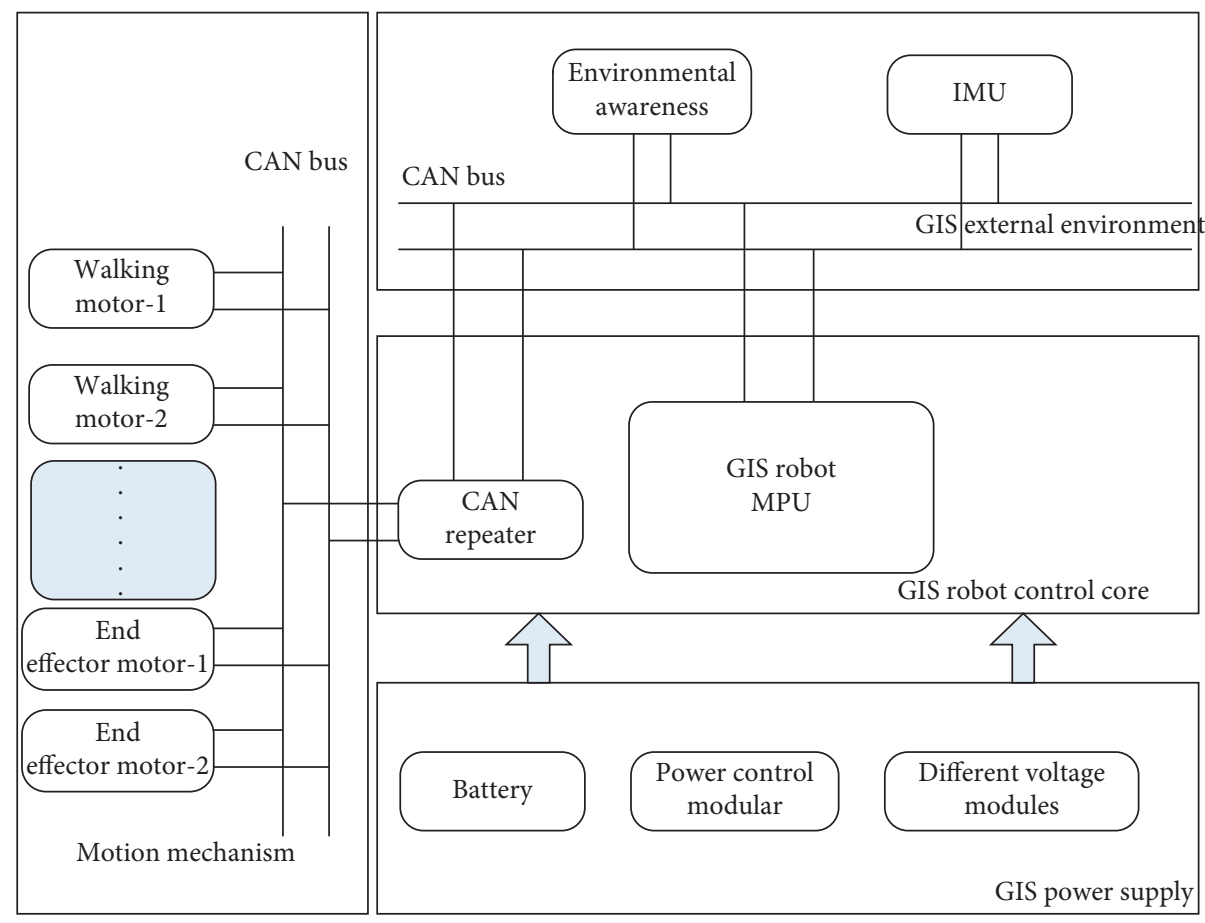

FIGURE 6: GIS inspection robot controller structure diagram.

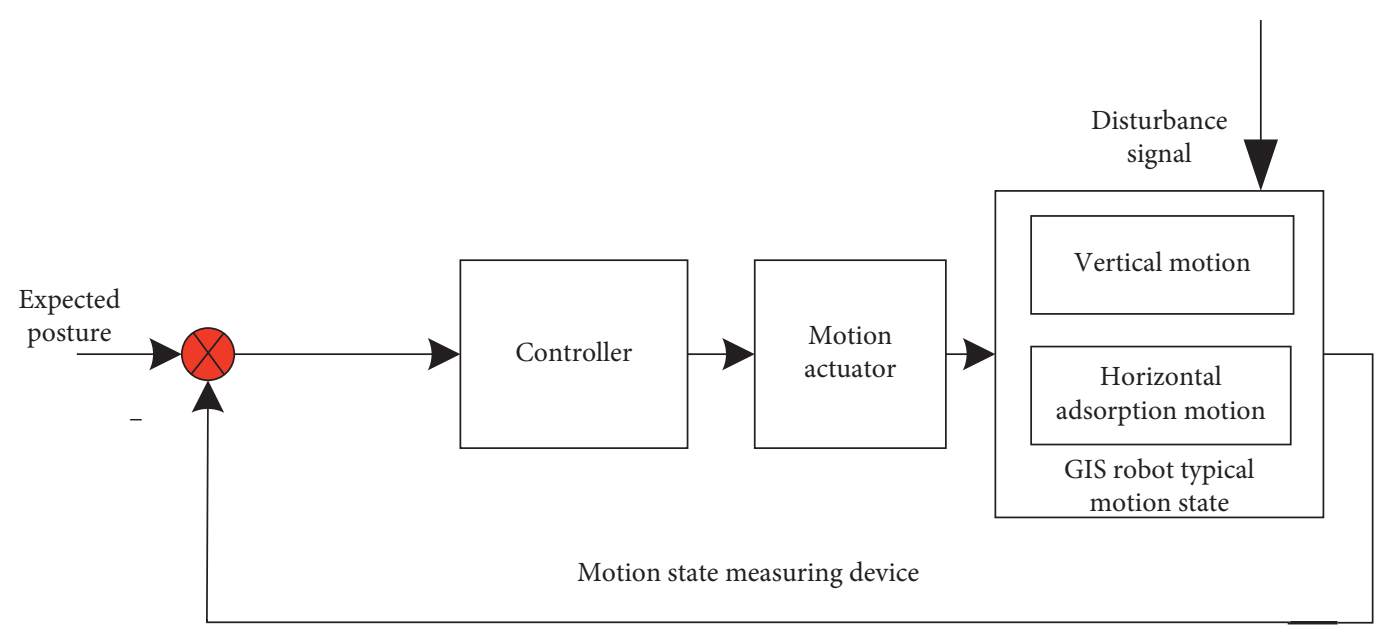

FIGURE 7: GIS inspection robot control system structure diagram.

regulator, and the whole motion control process can be divided into three parts. First of all, the environment-aware module, the IMU module, and the CAN repeater module can get the actual attitude information of the robot and take the information of the robot planning path stored in the MPU as the input value of the robot path planning. The MPU then obtains the coordinate values required by the robot so as to reach the destination according to the input value and the preset program and then obtains the corner information of the wheel under each coordinate value according to the motion inverse solution. Finally, the MPU transmits this information to the CAN repeater which completes the drive of the motor according to this information and finally realizes the motion control of the GIS robot. In addition, motion control needs to consider the influence of error as external information to participate in the closed-loop control of robot motion.

\section{GIS Inspection Robot Motion Modeling and Robust Stability Control}

4.1. Chassis Surface Motion Realization and Stability Analysis. Robot kinematics is an important theory that analyzes the relationship between the motion posture and joint variables of the robot manipulator in the space coordinate system. It is the theoretical basis of the GIS robot inspection control of the operation manipulator; equation (1) is homogeneous transformation matrix; the space posture of the manipulator 
can be obtained through the stacking calculation of the homogeneous transformation matrix as shown in equation (2); the posture of the manipulator in the base coordinate system is given as equation (3); equations (2) and (3) can be used to establish an equation relationship to solve the kinematics forward and inverse solutions of the robot manipulator and end effector.

$$
\begin{aligned}
{ }_{i}^{i-1} T & =\left[\begin{array}{cccc}
\cos \theta_{i} & -\sin \theta_{i} \cos \alpha_{i} & \sin \theta_{i} \sin \alpha_{i} & a_{i} \cos \theta_{i} \\
\sin \theta_{i} & \cos \theta_{i} \cos \alpha_{i} & -\cos \theta_{i} \sin \alpha_{i} & a_{i} \sin \theta_{i} \\
0 & \sin \alpha_{i} & \cos \alpha_{i} & d_{i} \\
0 & 0 & 0 & 1
\end{array}\right] \\
{ }_{n}^{0} T & ={ }_{1}^{0} T \cdot{ }_{2}^{1} T \cdots \cdot{ }_{n}^{n-1} T=\prod_{i=1}^{n}{ }_{i}^{i-1} T \\
{ }_{n}^{0} T & =\left[\begin{array}{cc}
R_{n}^{0} & P \\
0 & 1
\end{array}\right]=\left[\begin{array}{cccc}
n_{x} & o_{x} & a_{x} & P_{x} \\
n_{y} & o_{y} & a_{y} & P_{y} \\
n_{z} & o_{z} & a_{z} & P_{z} \\
0 & 0 & 0 & 1
\end{array}\right] .
\end{aligned}
$$

Chassis walking is the basis for the robot to complete GIS equipment inspection functions, the right chassis motion system designed in this paper needs to achieve way motion on the surface; Figure 8 is the calculation diagram of the chassis in the plane movement, and its motion inverse solution in plane motion is given in the following equation:

$$
\left[\begin{array}{l}
v_{1} \\
v_{2} \\
v_{3} \\
v_{4}
\end{array}\right]=\left[\begin{array}{ccc}
-\cos \alpha-\sin \alpha & -\cos \alpha+\sin \alpha & m+n \\
-\cos \alpha+\sin \alpha & -\cos \alpha-\sin \alpha & m+n \\
\cos \alpha+\sin \alpha & \cos \alpha-\sin \alpha & m+n \\
\cos \alpha-\sin \alpha & \cos \alpha+\sin \alpha & m+n
\end{array}\right]\left[\begin{array}{c}
v_{x} \\
v_{y} \\
\omega
\end{array}\right] \text {. }
$$

In equation (4), $v_{1}, v_{2}, v_{3}, v_{4}$ is the speed of three wheels, $\omega$ is the rotation angle speed, and $v_{x}, v_{y}$ is the speed in the body coordinate system, that is, the relative speed, because the chassis speed performance is independent of the attitude in the world coordinate system, so here is a simplified operation: take the body coordinate system and the world coordinate system $X, Y$ direction rematch; $m, n$ is the $x$ and $y$ distance from the center of rotation to the axis of the wheel which is half the length and width of the chassis; $\theta=45^{\circ}$ is the angle between the axle and the $x$-axis. When moving on a surface, considering that the robot only needs to maintain a definite attitude when moving on the surface, the forward- and crossshifting in the pipe can be achieved by the characteristics of the wheel chassis, so the angle velocity $\omega$ is constantly 0 , which brings convenience to the solution of surface motion. Since the deformation of the chassis does not affect the change of the attitude angle $\alpha$ and it is expressed in equation (1) and only changes the wheel base, the motion model of the GIS inspection robot when climbing on the surface is consistent with the plane motion model.

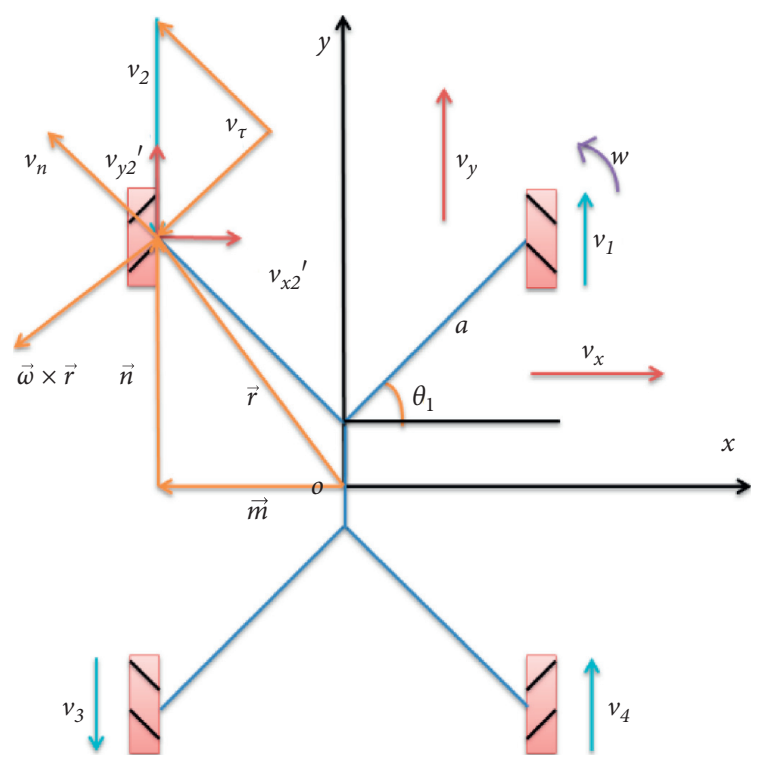

Figure 8: Map of the wheel chassis plane.

4.2. Analysis and Simulation of the Characteristics of Robot Climbing. GIS inspection robot in the three-dimensional wall climbing will affect the stability and flexibility of motion due to changes in the direction of gravity; in this section, through the up and down analysis on vertical wall and cantilever surface in three situations, their own acceleration curve is obtained and the GIS inspection robot start-up momentary acceleration changes and motion stability are analyzed. GIS inspection robot climbing schematic is shown in Figure 9.

In the analysis of the robot motion process, three typical motion processes have been analyzed, which are moving up the wall, moving down the wall, and moving cantilever on the wall. In order to facilitate the analysis, the physical amount involved in the motion process can be simplified, the GIS robot adsorption force in the condition of the three attitudes can be provided by the Halbach magnetic array, and the adsorption force is uniform and the motor drive force is continuous. Based on the above hypothesis, it can be obtained that when the GIS inspection robot moves up the vertical wall, its motion equation is equation (5), when the robot moves down the vertical wall, its motion equation is equation (6), when the robot moves along the horizontal suspension wall, its motion equation is equation (7), and the GIS robot friction force when moving on the wall is equation (8):

$$
\begin{aligned}
M \cdot \ddot{y} & =F_{\text {drive } 1}-c \cdot F_{f}-G, \\
M \cdot \ddot{y} & =F_{\text {drive } 2}-c \cdot \dot{y}-F_{f}+G, \\
M \cdot \ddot{x} & =F_{\text {drive } 3}-c \cdot \dot{x}-F_{f}, \\
F_{f} & =\mu \sum N_{i} .
\end{aligned}
$$

In the above equations, $M$ represents the total mass of the robot $(\mathrm{kg}), F_{\text {drivel }}$ represents the total driving force of the wheel motor when the robot moves upward, $x$ represents the 


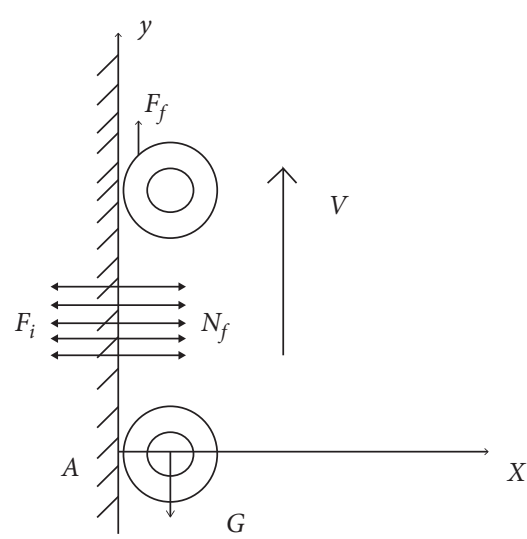

(a)

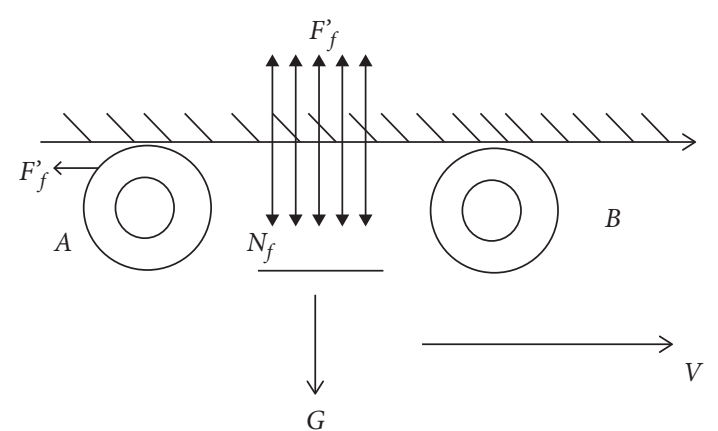

(b)

Figure 9: Analysis schematic of climbing characteristics. (a) Vertical schematic; (b) cantilever schematic.

acceleration of the robot, $x$ indicates the speed of the robot, $G$ represents the gravity of the robot system, $F_{f}$ represents the total sliding friction of the robot, $C$ represents the damping coefficient obtained by a combination of various factors when the robot moves, unit is $\mathrm{N} / \mathrm{ms}, \mu$ represents the coefficient of friction between the $\mathrm{McNem}$ wheel and the wall, $F_{\text {drive2 }}$ represents the total motor-to-wheel drive when the robot moves down, and $F_{\text {drive3 }}$ represents the total motor-to-wheel drive when the robot moves downwards, which represents the acceleration of the robot when moving horizontally and the speed of the robot when moving horizontally.

\section{Simulation and Field Operation Experiment}

5.1. Simulation Experiments and Analysis. In this paper, ANSYS Maxwell electromagnetic simulation software has been used to simulate the magnet adsorption unit; the magnetic force size of the magnet and the adsorption wall surface can be obtained under different gaps. The simulation parameters are selected as follows: steel-1010 sheet with a $5 \mathrm{~mm}$ thickness is placed on each side of the double magnetic adsorption arrays, and the magnetic magnetization direction is specified in accordance with the above rules and the distance between the magnetic adsorption module and the wall surface is used as a sweep parameter, with a range of $3 \mathrm{~mm}$ to $8 \mathrm{~mm}$. Finally, the adsorption force of the magnetic adsorption module on the wall surface can be concluded, and the simulation data in Figure 10 can be obtained in Table 1; it can be seen that the magnetic force generated by the ordinary array on both sides of its adsorption unit is exactly the same in the simulation environment; the Halbach array almost eliminates the magnetic field on one side and the magnetic force generated on the other side has greatly increased, about twice that of the ordinary array.

Through the above analysis, we can get the mathematical relationship model between the adsorption force, friction force, robot total mass, and motor total driving moment of Halbach magnetic array in three cases. The robot motion instantaneous acceleration and stability analysis can be carried out by solving the above system and can be simulated using MATLAB/Simulink software; the structure of the Simulink block is shown in Figure 11.

Through running the Simulink simulation, we can obtain the acceleration curve of the robot in three cases, as shown in Figure 12, where the robot starts with a momentary impact, and when the robot moves up the vertical wall, the instantaneous acceleration can reach $7 \mathrm{~m} / \mathrm{s}^{2}$, at which point the robot is subjected to the greatest instantaneous impact, and, again, the momentary acceleration of the downward movement can reach $6 \mathrm{~m} / \mathrm{s}^{2}$, and the instantaneous acceleration along the horizontal cantilever surface is $4 \mathrm{~m} / \mathrm{s}^{2}$. With the increase of start-up time, the robot is subject to self-vibration caused by the discontinuous design of the outer roller of the Mecanum wheel; the robot motion acceleration has rapidly reduced and eventually became zero, at which point the robot will begin to move at a constant speed; that is, the robot will soon reach a stable state, which indicates that the GIS climbing robot system designed in this paper has a certain degree of motion stability.

5.2. GIS Robot Climbing Performance Experiments and Analysis. In this section, the adsorption experiments have been conducted in different environments for the GIS robot prototype, which have been tested in different thicknesses and different wall environments; the gap between the wall surface and the magnetic array was $2 \mathrm{~mm}$ during the experiment. Figure 13 is the field operation experiment, with the four typical motion states, respectively, in Figures 13(a)13(d); in GIS equipment steel pipe suspension surface and vertical wall surface walking experiments, the experimental plane is relatively flat and barrier-free; the robot runs smoothly; overall, GIS inspection robot on different wall surface adaptability and adsorption force meets the climbing requirements of GIS equipment maintenance process; in this simulated working environment, its wall thickness is much 


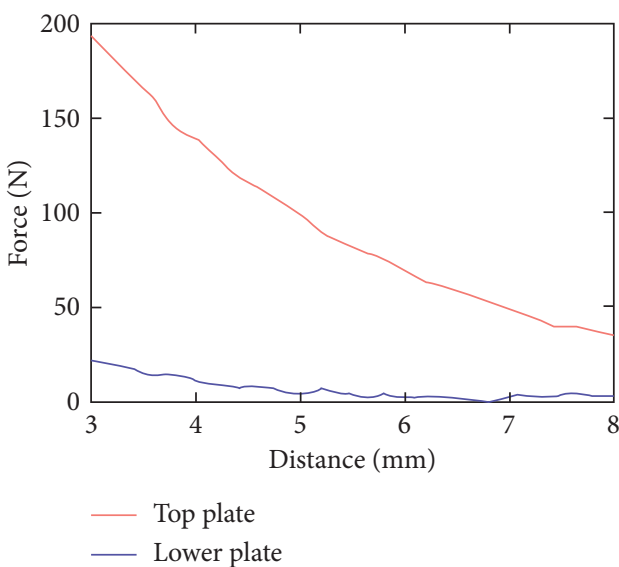

(a)

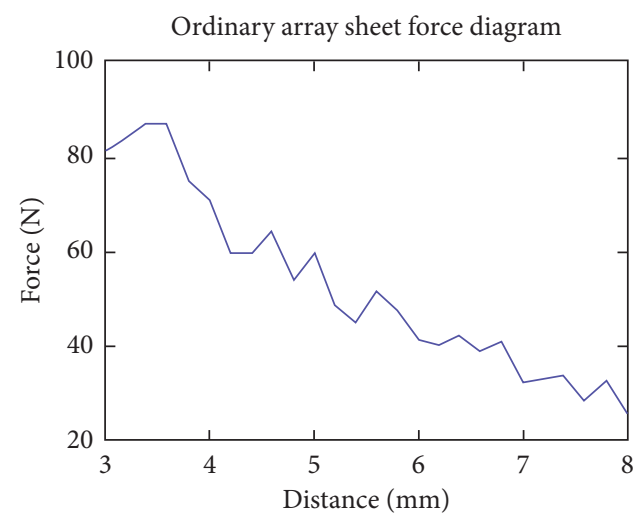

(b)

Figure 10: The force contrast of two magnetic arrays on wall surfaces. (a) Force diagram of Halbach array sheets; (b) force diagram of ordinary array sheets.

TABle 1: Force data of Halbach array and ordinary array sheets.

\begin{tabular}{lccc}
\hline \multirow{2}{*}{ Force } & \multicolumn{2}{c}{ Halbach array sheets } & Ordinary array sheets $(\mathrm{N})$ \\
& Top plane $(\mathrm{N})$ & Lower plane $(\mathrm{N})$ & 86 \\
Max & 192 & 22 & 12 \\
\hline Min & 38 & 2 & 12 \\
\hline
\end{tabular}

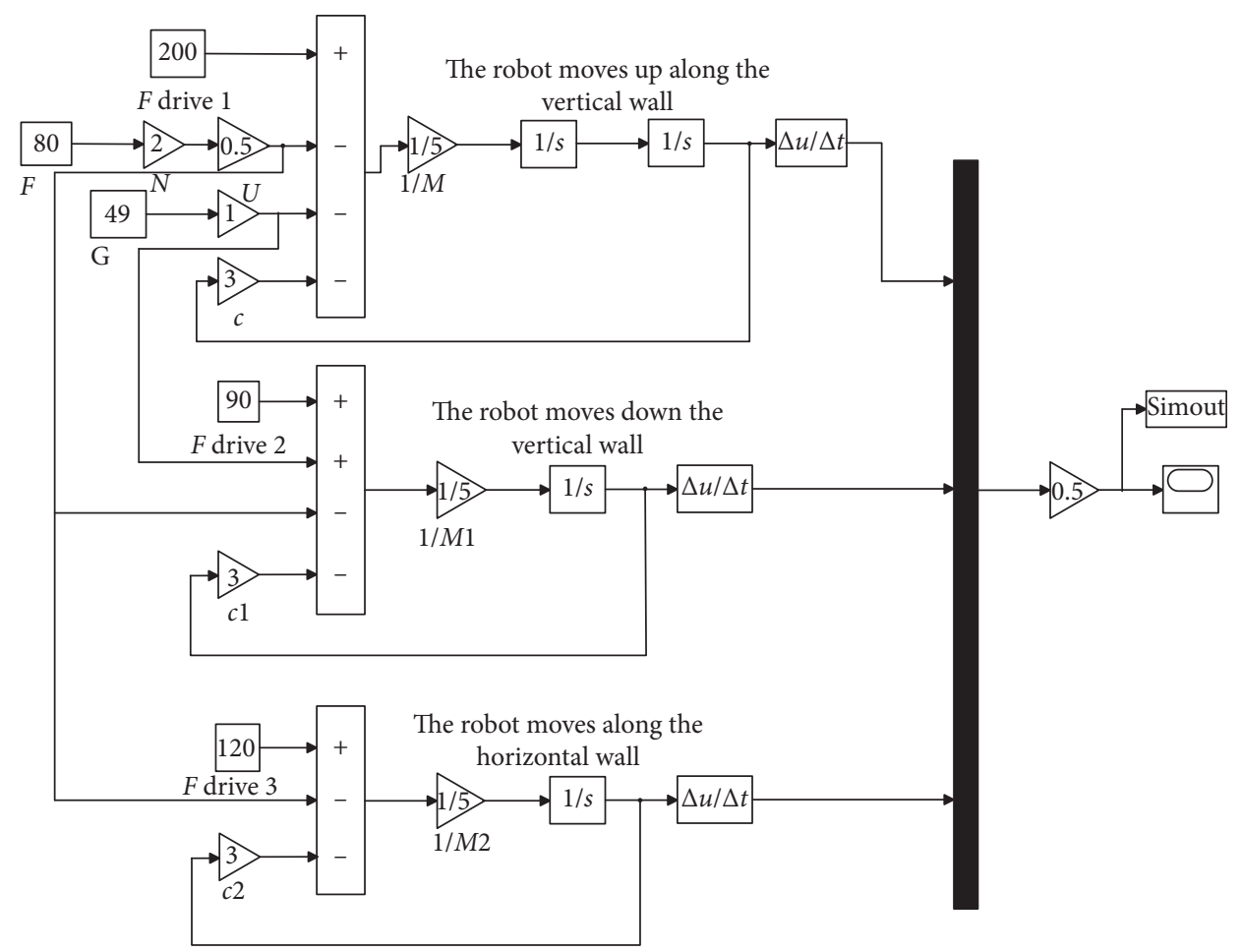

FiguRE 11: GIS robot control system based on MATLAB/Simulink simulation.

larger than the tin cabinet wall; therefore, the GIS inspection robot can also meet the actual GIS equipment inspection and maintenance needs.
5.3. GIS Robot Field Operation Experiments and Analysis. Selection of the typical GIS equipment was under the jurisdiction of the live working operation center, State Grid 


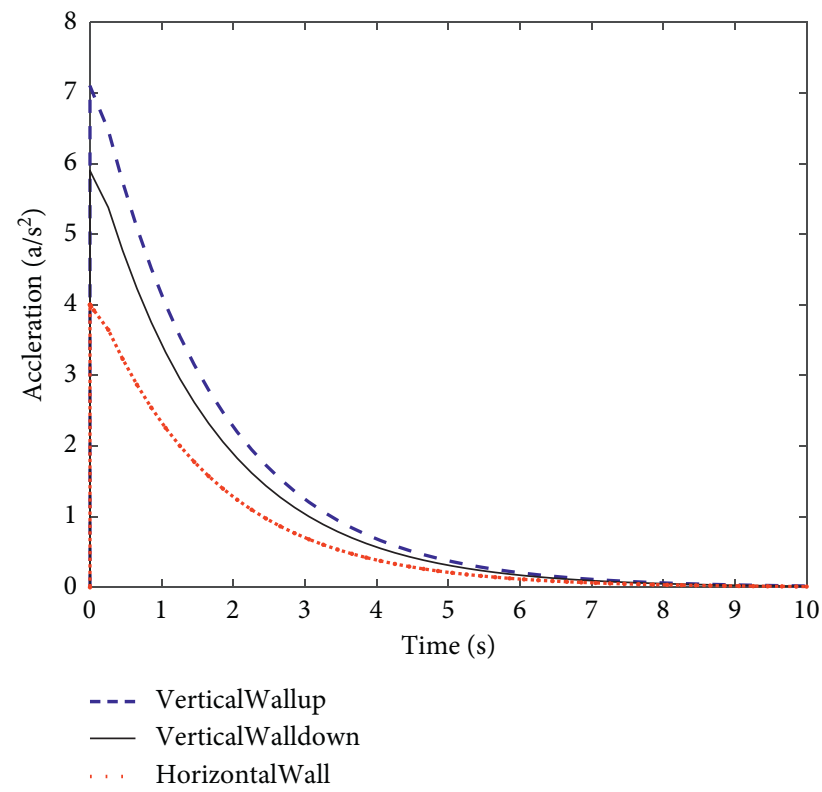

FIGURE 12: MATLAB/Simulink simulation results.

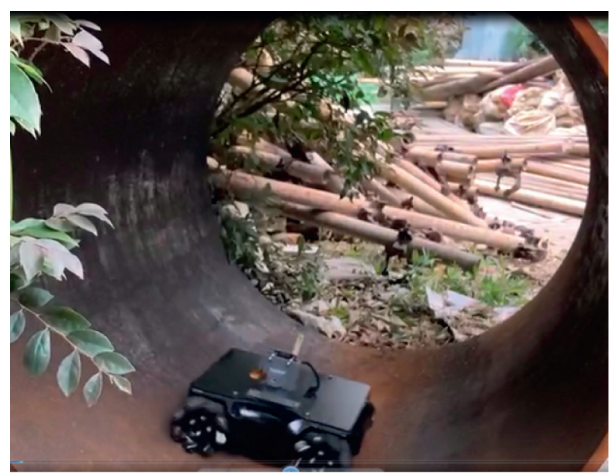

(a)

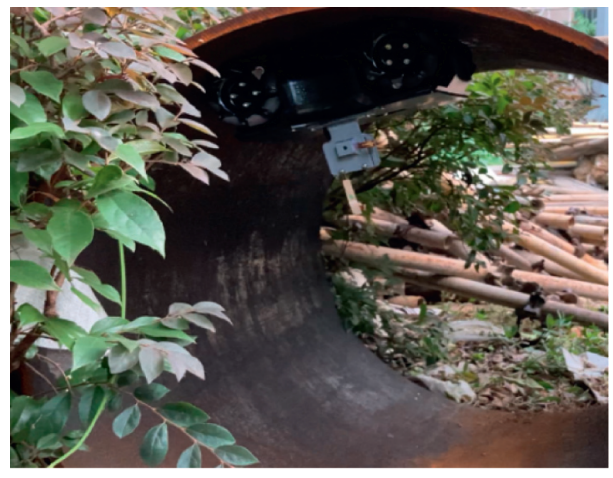

(c)

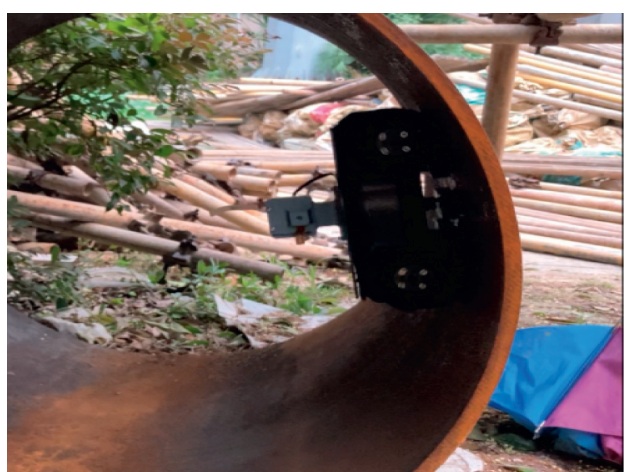

(b)

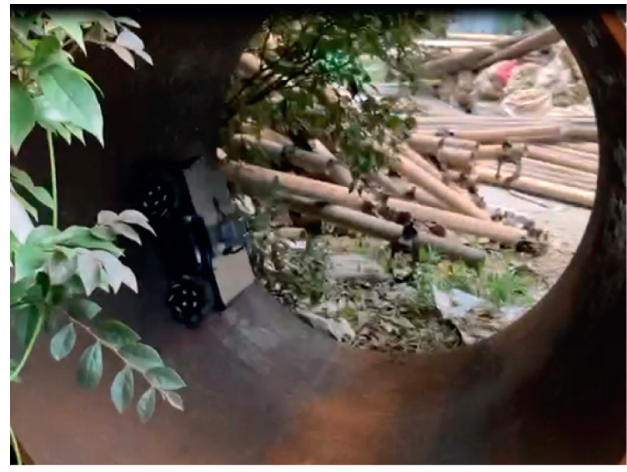

(d)

FIGURE 13: GIS inspection robot climbing performance experiment in simulated environment. (a) Horizontal (initial state), (b) right vertical wall, (c) left vertical wall, and (d) return to initial state.

Hunan Electric Power Co., Ltd.; the inspection operation field experiment has been carried out; Figure 14 shows the movement and inspection status of the GIS inspection robot inside the GIS equipment. It can be seen from the experimental results that the robot can move freely inside the GIS equipment and can realize maintenance operations at any position. Through the operation experiment, the main performance indexes of GIS inspection robot can be obtained and are shown in Table 2; regarding the structure, the robot is small in size and light in weight, which is suitable for 


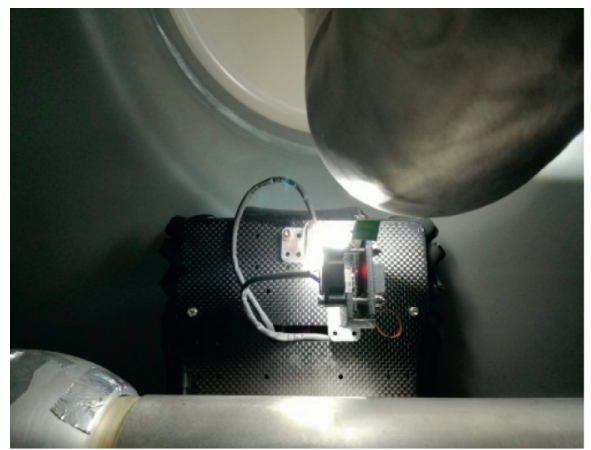

(a)

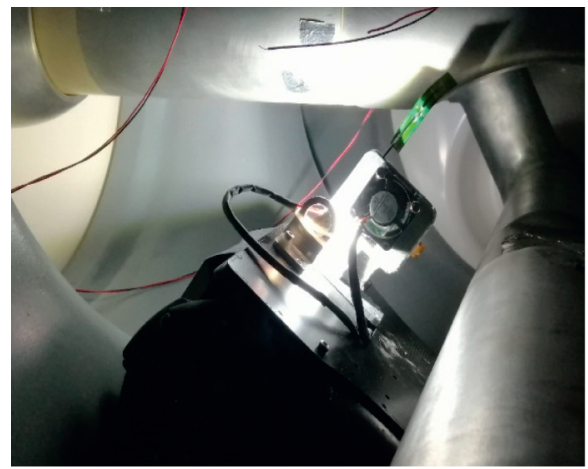

(c)

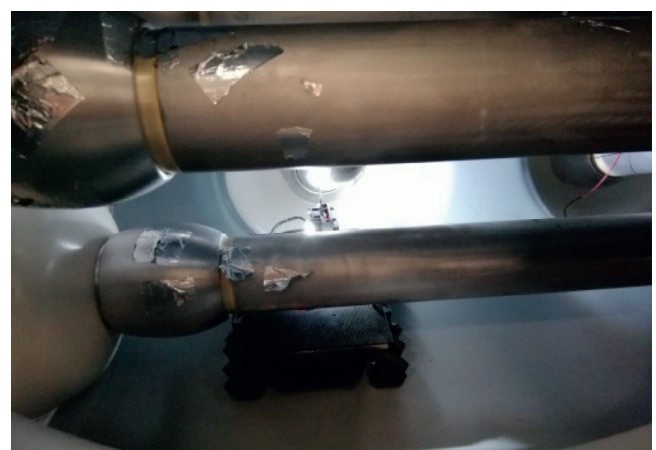

(b)

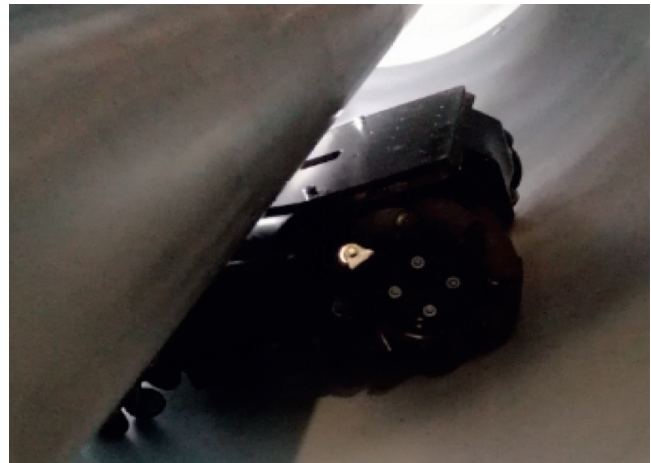

(d)

FIGURE 14: GIS inspection robot field operation experiment. (a) GIS robot field operation state 1, (b) GIS robot field operation state 2, (c) GIS robot field operation state 3 , and (d) GIS robot field operation state 4.

TABLE 2: The main performance indexes of GIS inspection robot.

\begin{tabular}{lcc}
\hline Main performance indexes & Performance indexes values & $\begin{array}{c}\text { Operation process status (whether meeting } \\
\text { the operation requirements) }\end{array}$ \\
\hline Robot operational efficiency (inspection speed) & $3 \mathrm{~m} / 2 \mathrm{~min}$ & OK \\
Main operation objects positioning accuracy & $1 \mathrm{~mm}$ & OK \\
Man-machine interaction communication distance & $50 \mathrm{~m}$ & OK \\
Overall size of GIS robot & $221 \times 188 \times 154 \mathrm{~mm}$ & OK \\
Total mass of GIS robot & $3.3 \mathrm{~kg}$ & OK \\
\hline
\end{tabular}

maintenance in GIS equipment; regarding the other aspects, compared with manual operation, GIS robot inspection efficiency is high, obstacle fault location is more accurate, and remote wireless communication control can be achieved; therefore, the GIS inspection robot developed in this paper has strong engineering practicability in the power system.

\section{Conclusions and Future Work}

\subsection{Conclusions}

(1) A basic configuration of GIS inspection robot based on adsorption capability has been proposed, which can climb along the GIS equipment suspension surface and vertical surface, as well as a method for motion planning of maintenance and inspection operations without blind spots within GIS equipment
(2) The typical state force model of GIS inspection robot has been established in the process of internal inspection of GIS equipment, the force model of vertical and overhang surface has been established, the characteristics of its magic have been analyzed, and the robust stability control method of GIS inspection robot has been proposed

(3) An embedded integrated control system architecture and physical implementation method of GIS inspection robot have been proposed, a physical prototype system of GIS inspection robot has been developed, and the feasibility and effectiveness of GIS inspection robot operation have been verified by simulation and field experiments in complex microgrid networks

6.2. Future Work. GIS inspection robot replaces manual operation to complete the task in the narrow working space 
of GIS equipment; the main research directions in the future include two parts: one is how to locate the obstacles and work objects independently in the narrow working space of GIS equipment, and the other is how to locate the obstacles and work objects without collision in the narrow working space of GIS equipment; therefore, in the complex operation environment, especially in the disturbance motivation, how to stabilize the robot's operation, that is, the robustness of the robot's operation, will be the focus of the follow-up research.

\section{Data Availability}

The data used to support the findings of this study are included within the article.

\section{Conflicts of Interest}

The authors declare that they have no conflicts of interest.

\section{Acknowledgments}

This work was supported by the Science and Technology Project of Hunan Electric Power Company (B116A320001D-16-16) and 2020 Opening Fund for Hubei Key Laboratory of Digital Textile Equipment (DTL2020010).

\section{References}

[1] K.-H. Seok and Y. S. Kim, "A state of the art of power transmission line maintenance robots," Journal of Electrical Engineering and Technology, vol. 11, no. 5, pp. 1412-1422, 2016.

[2] X. Yue, H. Wang, and Y. Jiang, "A novel $110 \mathrm{kV}$ power line inspection robot and its climbing ability analysis," International Journal of Advanced Robotic Systems, vol. 14, no. 3, pp. 1-10, 2017.

[3] C. M. Shruthi, A. P. Sudheer, and M. L. Joy, "Optimal crossing and control of mobile dual-arm robot through tension towers by using fuzzy and Newton barrier method," Journal of the Brazilian Society of Mechanical Sciences and Engineering, vol. 41, no. 6, pp. 245-270, 2019.

[4] C. Antonini, M. Innocenti, T. Horn, M. Marengo, and A. Amirfazli, "Understanding the effect of superhydrophobic coatings on energy reduction in anti-icing systems," Cold Regions Science and Technology, vol. 67, no. 1-2, pp. 58-67, 2011.

[5] S. Farhadi, M. Farzaneh, and S. A. Kulinich, "Anti-icing performance of superhydrophobic surfaces," Applied Surface Science, vol. 257, no. 14, pp. 6264-6269, 2011.

[6] S. A. Kulinich and M. Farzaneh, "On ice-releasing properties of rough hydrophobic coatings," Cold Regions Science and Technology, vol. 65, no. 1, pp. 60-64, 2011.

[7] Y. Wang, J. Yan, Q. Sun, J. Li, and Z. Yang, "A MobileNets convolutional neural network for GIS partial discharge pattern recognition in the ubiquitous power internet of things context: optimization, comparison, and application," IEEE Access, vol. 7, pp. 150226-150236, 2019.

[8] X. Fu, H. Feng, and X. Gao, "UAV mobile ground target pursuit algorithm," Journal of Intelligent \& Robotic Systems, vol. 68, no. 3-4, pp. 359-371, 2012.

[9] W. Wang, G.-P. Wu, Y.-C. Bai et al., "Hand-eye-vision based control for an inspection robot's autonomous line grasping,"
Journal of Central South University, vol. 21, no. 6, pp. 22162227, 2014.

[10] E. Peri and A. Tal, "A sustainable way forward for wind power: assessing turbines' environmental impacts using a holistic GIS analysis," Applied Energy, vol. 279, Article ID 115829, 2020.

[11] J.-Y. Park, J.-K. Lee, B.-H. Cho, and K.-Y. Oh, "An inspection robot for live-line suspension insulator strings in $345 \mathrm{kV}$ power lines," IEEE Transactions on Power Delivery, vol. 27, no. 2, pp. 632-639, 2012.

[12] J. Lai, X. Lu, X. Yu, and A. Monti, "Cluster-oriented distributed cooperative control for multiple ac microgrids," IEEE Transactions on Industrial Informatics, vol. 15, no. 11, pp. 5906-5918, 2019.

[13] J. Lai, X. Lu, X. Yu, and A. Monti, "Stochastic distributed secondary control for ac microgrids via event-triggered communication," IEEE Transactions on Smart Grid, vol. 11, no. 4, pp. 2746-2759, 2020.

[14] T. Q. Ngo, Y. N. Wang, and T. L. Mai, "Robust adaptive neural-fuzzy network tracking control for robot manipulator," International Journal of Computers Communications \& Control, vol. 7, no. 2, pp. 341-352, 2016.

[15] R. Miller, F. Abbasi, and J. Mohammadpour, "Power line robotic device for overhead line inspection and maintenance," Industrial Robot: An International Journal, vol. 44, no. 1, pp. 75-84, 2017.

[16] V. Alakshendra and S. S. Chiddarwar, "Simultaneous balancing and trajectory tracking control for an omnidirectional mobile robot with a cylinder using switching between two robust controllers," International Journal of Advanced Robotic Systems, vol. 14, no. 6, pp. 1-12, 2017.

[17] G. Mamani, J. Becedas, and V. Feliu, "Sliding mode tracking control of a very lightweight single-link flexible robot robust to payload changes and motor friction," Journal of Vibration and Control, vol. 18, no. 8, pp. 1141-1155, 2012.

[18] N. Xu and T. Ishihara, "Analytical formulae for wind turbine tower loading in the parked condition by using quasi-steady analysis," Wind Engineering, vol. 38, no. 3, pp. 291-309, 2014.

[19] Y. Luo, H. Liu, H. Xue, and K. Lin, "Large-eddy simulation evaluation of wind loads on a high-rise building based on the multiscale synthetic eddy method," Advances in Structural Engineering, vol. 22, no. 4, pp. 997-1006, 2019.

[20] J. Lai and X. Lu, "Nonlinear mean-square power sharing control for ac microgrids under distributed event detection," IEEE Transactions on Industrial Informatics, vol. 17, no. 1, p. 219, 2021. 